\title{
Evaluation of New Fungicides Molecules against Leaf Blight of Chrysanthemum caused by Alternaria spp.
}

\author{
U. Divyajyothi ${ }^{*}{ }^{*}$ Suresh D. Ekabote $^{3}$, H. Narayanswamy ${ }^{4}$, \\ P. Narayanaswamy ${ }^{5}$, H. Ravindra ${ }^{6}$ and K.M. Satish ${ }^{2}$
}

${ }^{1}$ College of Agriculture, ${ }^{2}$ Department of Plant Biotechnology, Shivamogga University of

Agricultural and Horticultural Sciences, Shivamogga -580 005, India

${ }^{3}$ Department of Crop Protection, College of Horticulture, Hiriyur, University of Agricultural and Horticultural Sciences, Shivamogga -580 005, India

${ }^{4}$ Department of Plant Pathology, ${ }^{5}$ UAHS, ${ }^{6}$ AICRP $(N)$, Shivamogga, India

*Corresponding author

\section{A B S T R A C T}

\begin{tabular}{|l|}
\hline Ke y w or d s \\
$\begin{array}{l}\text { Chrysanthemum, } \\
\text { Alternaria, Leaf } \\
\text { blight, Fungicides }\end{array}$ \\
\hline Article Info \\
\hline $\begin{array}{l}\text { Accepted: } \\
\text { 26 June } 2018 \\
\text { Available Online: } \\
\text { 10 July } 2018\end{array}$ \\
\hline
\end{tabular}

\section{Introduction}

Chrysanthemum (Dendranthema grandiflora Tzvelev.) is a multi-use flower crop belonging to Asteraceae family, and gaining more popularity as a cut flower, loose flowers and pot plant. Chrysanthemum is commonly known as Queen of East produces very attractive flowers of different shape, size and colours. It is an important commercial flower next to rose in the international florists trade and grown throughout the world (Kher, 1990).
Alternaria leaf blight is one of the most important foliar disease of chrysanthemum. It has become major constraint in chrysanthemum growing areas. The fungicides were evaluated under field conditions at College of Horticulture, Hiriyur, during kharif 2016 and 2017. Five sprays were given at 60, 75, 90, 105 and 120 DAS. Two years pooled results revealed that tebuconazole $(0.1 \%)$ significantly higher per cent disease control (PDC) of 45.20 which was followed by tebuconazole+ trifloxystrobin (43.07) and propiconazole (40.67). Similarly, the maximum yield of 8.78 t/ha was recorded in tebuconazole $(0.1 \%)$ which was followed by tebuconazole+ trifloxystrobin $(8.22 \mathrm{t} / \mathrm{ha})$ and propiconazole $(8.11 \mathrm{t} / \mathrm{ha})$ respectively. However, tebuconazole recorded highest benefit:cost of 4.13 followed by hexaconazole 3.92 remained as next best fungicide.

In India, chrysanthemum occupied a place of pride, both as commercial loose flower crop and is commercially cultivated in major chrysanthemum growing states viz., Karnataka, Tamil Nadu, Maharashtra, Rajasthan, Madhya Pradesh and Bihar. In India, chrysanthemum is grown in an area of 0.16 lakh ha with the production of 1799.70 lakh tonnes of loose flowers and 57.1 lakh tonnes of cut flowers (NHB 2014). In Karnataka Chitradurga, Tumkuru, Kolar, Bengaluru, Chikkaballapur, Doddaballapur are 
the major growing districts and it is grown mainly for loose flower purpose with leading area of 4429 ha and production of 54092 Metric tonnes. The productivity is 12.21 MT/ha (Anon., 2016). There has been a regular demand for chrysanthemum flowers throughout the year in our country. Hence, there is a great potential for production of chrysanthemum on a commercial scale in India. However, it is difficult to get good quality exportable blooms, higher yields and long lasting post harvest life of the cultivars under open conditions. The most important factors responsible for the threatened production of chrysanthemum flowers yield is by many diseases such as Alternaria leaf blight, Fusarium wilt, Septorial leaf spot, Ray speck disease, Pythium rot, Chrysanthemum rust, Bacterial blight, viruse (viriod) and Nematode (Schmidt, 1958; Alfieri, 1968; Strider, 1985; Cook, 2001; Nishi et al., 2009; Luong et al., 2010) and non availability of leading varieties which are resistant to biotic and abiotic stresses and imbalance use of fertilizers.

Among the foliar diseases Alternaria leaf blight caused by Alternaria spp. is one of the most destructive diseases, commonly prevailing in almost all chrysanthemum growing pockets of India, which causes heavy losses under field as well as under market conditions. Since there are no sources of resistance available for the cultivation, farmers are largely depended on use of fungicides to manage this disease. Looking in to these bottle necks and also to tackle the problem of fungicidal resistance the present investigation was undertaken to identify the new effective molecules which derive maximum benefit to the farmers.

\section{Materials and Methods}

Field experiment was conducted at College of Horticulture, Hiriyur during kharif 2016-17 and $2017-18$ in relation to manage the leaf blight of chrysanthemum. The local variety was grown as per packages of practices for higher yields. The trial was laid out in Randomized Block Design (RBD) with three replications with plot $3 \times 2 \mathrm{~m}$ size. The treatments are as below: $\mathrm{T}_{1}$-Tebuconazole, $\mathrm{T}_{2-}$ Tebuconazole $50 \%+$ trifloxystrobin $25 \% \mathrm{WG}$, $\mathrm{T}_{3}$-Azoxystrobin $4.8 \%$ + Chlorothalonil $40 \%$, $\mathrm{T}_{4}$-Chlorothalonil $75 \% \mathrm{WP}, \mathrm{T}_{5}$-Azoxystrobin $250 \% \quad \mathrm{SC}, \mathrm{T}_{6}$-Propineb $70 \% \quad \mathrm{WP}, \mathrm{T}_{7^{-}}$ Mancozeb 70\%WP, $\mathrm{T}_{8}$-Hexaconazole 5\% EC, $\mathrm{T}_{9}$-Propiconazole $25 \% \mathrm{EC}, \mathrm{T}_{10}$-Difenconzole $25 \% \mathrm{EC}$ and $\mathrm{T}_{11}$-Control. The spray was given out given at 60,75, 90, 105 and 120 Days. The Per cent disease index (PDI) was computed by selecting five plants in each treatment at random and recording PDI as per 0-5 scale is given by (Sikdar and Krishnaswami, 1980; Mridha et al., 2007; Ghosh et al., 2009) and description given below in Table A.

The recorded grade values were converted into Percent Disease Index (PDI) by using following formula proposed by Wheeler (1969).

Per cent disease index $(\mathrm{PDI})=$

Sum of the individual disease ratings $\times 100$

Number of leaves observed $\times$ Maximum disease grade

The observation on flower yield expressed in terms of $t /$ ha was also recorded. The economic analysis was done by working out net income; benefit cost ratio was also worked out taking into account total cost of cultivation in control and additional cost for fungicides and their sprays. The data was statistically analyzed after suitable transformations.

\section{Results and Discussion}

A field experiment was conducted at College of Horticulture, Hiriyur as explained in 
'material methods' to find out the best chemical for management of leaf blight of chrysanthemum during the kharif 2016 and 2017. Totally five spray were given at 15 day intervals starting from initiation of disease. The Observations were recorded at 15 days after spray (DAS) by using 0-5 scale and converted into per cent disease index (PDI) using the formula given by Wheeler (1969) and calculated yield was statistically analyzed, data were presented in the Table 1 and 2 .

During kharif 2016 PDI before treatment imposition was non-significant and all the treatments remained on par or almost uniform with each other upto 60 days. On the contrary treatments started differed significantly at 75 , 90, 105 and 120 DAS over untreated control are presented in Table 1. At 75 DAS, tebuconazole recorded least PDI (10.40) was found on par with tebuconazole + trifloxystrobin (13.04 PDI) followed by propiconazole (15.20 PDI), difenconzole (17.60 PDI) and hexaconazole (18.13 PDI). Highest PDI was recorded at Untreated control (28.53 PDI) followed by chlorothalonil 75\%WP (27.47 PDI) respectively.

At 90, 105, 120 DAS also the trend was same, tebuconazole recorded least PDI of 12.27, 13.33 and 13.33 respectively, and it was on par with tebuconazole + trifloxystrobin (14.40, 15.47 and 16.00 respectively) which were followed difenconazole and hexaconazole. Maximum PDI was recorded in untreated control. During the second year spray (2017) there is no much significant differences among the treatments imposed with respect to the reduction of disease compare to first season. tebuconazole recorded least PDI at 60, 75, 90, 105 and 120 DAS $(11.20,14.13,18.13,20.53$ and 25.33 PDI respectively) which was on par with tebuconazole + trifloxystrobin (8.27, $14.67,19.20, \quad 21.60$ and 28.00 PDI respectively) which were followed by propiconazole $(9.87,14.93,19.73,22.93$ and 30.67 PDI respectively).

Pooled analysis revealed that, there is significant difference between treatments with respect to PDI over untreated treatments which are presented in Table 1.

At 75 DAS, tebuconazole recorded least PDI (12.27) was found on par with tebuconazole + trifloxystrobin (13.87) which were followed by propiconazole (15.07 PDI), difenconzole (17.07 PDI) and hexaconazole (17.87 PDI). Highest PDI was recorded at Untreated control (33.73 PDI) followed by chlorothalonil (27.07 PDI).

At 90, 105, 120 DAS also the trend was same, tebuconazole recorded least PDI and was on par with tebuconazole + trifloxystrobin which were followed by propiconazole, difenconazole and hexaconazole. Maximum PDI was observed at untreated control.

Table.1a A Description of disease scale (0-5)

\begin{tabular}{|l|l|}
\hline Scale & Description \\
\hline $\mathbf{0}$ & No disease symptoms \\
\hline $\mathbf{1}$ & A few spots towards tip covering 10 per cent leaf area \\
\hline $\mathbf{2}$ & Several dark brown patches covering upto 20 per cent leaf area \\
\hline $\mathbf{3}$ & Several patches with paler outer zone covering upto 40 per cent leaf area \\
\hline $\mathbf{4}$ & Leaf blight covering upto 75 per cent leaf area or breaking of the leaves from centre \\
\hline $\mathbf{5}$ & Complete drying of the leaves or breaking of the leaves from centre \\
\hline
\end{tabular}


Table.1 Field efficacy of fungicides on Per cent Disease Index (PDI) of chrysanthemum cultivar Pache

\begin{tabular}{|c|c|c|c|c|c|c|c|c|c|c|c|c|c|c|c|}
\hline \multirow[t]{3}{*}{ Treatments } & \multicolumn{15}{|c|}{ PDI at Days after spray } \\
\hline & \multicolumn{3}{|c|}{60 Day } & \multicolumn{3}{|c|}{ 75Days } & \multicolumn{3}{|c|}{90 Days } & \multicolumn{3}{|c|}{ 105Days } & \multicolumn{3}{|c|}{ 120Days } \\
\hline & 2016 & 2017 & Pooled & 2016 & 2017 & Pooled & 2016 & 2017 & Pooled & 2016 & 2017 & Pooled & 2016 & 2017 & Pooled \\
\hline T1-Tebuconazole & $\begin{array}{c}9.60 \\
(18.06)^{*}\end{array}$ & $\begin{array}{c}11.20 \\
(19.56)\end{array}$ & $\begin{array}{l}10.40 \\
(18.82)\end{array}$ & $\begin{array}{c}10.40 \\
(18.82)\end{array}$ & $\begin{array}{c}14.13 \\
(22.09)\end{array}$ & $\begin{array}{l}12.27 \\
(20.51)\end{array}$ & $\begin{array}{c}12.27 \\
(20.51)\end{array}$ & $\begin{array}{c}18.13 \\
(25.22)\end{array}$ & $\begin{array}{l}15.20 \\
(22.96)\end{array}$ & $\begin{array}{c}13.33 \\
(21.43)\end{array}$ & $\begin{array}{l}20.53 \\
(26.96)\end{array}$ & $\begin{array}{l}16.93 \\
(24.31)\end{array}$ & $\begin{array}{c}14.40 \\
(22.31)\end{array}$ & $\begin{array}{l}25.33 \\
(30.24)\end{array}$ & $\begin{array}{c}19.87 \\
(26.48)\end{array}$ \\
\hline $\begin{array}{c}\text { T2-Tebuconazole } \\
50 \%+\text { trifloxystrobin } \\
25 \% \mathrm{WG}\end{array}$ & $\begin{array}{c}8.53 \\
(16.99)\end{array}$ & $\begin{array}{c}8.27 \\
(16.72)\end{array}$ & $\begin{array}{c}8.40 \\
(16.86)\end{array}$ & $\begin{array}{l}13.07 \\
(21.20)\end{array}$ & $\begin{array}{l}14.67 \\
(22.53)\end{array}$ & $\begin{array}{c}13.87 \\
(21.87)\end{array}$ & $\begin{array}{c}14.40 \\
(22.31)\end{array}$ & $\begin{array}{c}19.20 \\
(26.00)\end{array}$ & $\begin{array}{l}16.80 \\
(24.21)\end{array}$ & $\begin{array}{c}15.47 \\
(23.17)\end{array}$ & $\begin{array}{l}21.60 \\
(27.71)\end{array}$ & $\begin{array}{c}18.53 \\
(25.51)\end{array}$ & $\begin{array}{l}16.00 \\
(23.59)\end{array}$ & $\begin{array}{l}28.00 \\
(31.96)\end{array}$ & $\begin{array}{c}22.00 \\
(27.99)\end{array}$ \\
\hline $\begin{array}{l}\text { T3-Azoxystrobin } 4.8 \% \\
+ \text { Chlorothalonil } 40 \%\end{array}$ & $\begin{array}{c}8.27 \\
(16.72)\end{array}$ & $\begin{array}{c}9.07 \\
(17.53)\end{array}$ & $\begin{array}{c}8.67 \\
(17.13)\end{array}$ & $\begin{array}{l}20.27 \\
(26.77)\end{array}$ & $\begin{array}{c}17.33 \\
(24.62)\end{array}$ & $\begin{array}{c}18.80 \\
(25.71)\end{array}$ & $\begin{array}{l}24.00 \\
(29.35)\end{array}$ & $\begin{array}{c}25.87 \\
(30.59)\end{array}$ & $\begin{array}{c}24.93 \\
(29.97)\end{array}$ & $\begin{array}{c}28.53 \\
(32.30)\end{array}$ & $\begin{array}{l}29.87 \\
(33.14)\end{array}$ & $\begin{array}{l}29.20 \\
(32.73)\end{array}$ & $\begin{array}{c}30.93 \\
(33.81)\end{array}$ & $\begin{array}{c}38.13 \\
(38.15)\end{array}$ & $\begin{array}{c}34.53 \\
(36.01)\end{array}$ \\
\hline $\begin{array}{c}\text { T4-Chlorothalonil 75\% } \\
\text { WP }\end{array}$ & $\begin{array}{c}9.07 \\
(17.53)\end{array}$ & $\begin{array}{c}10.40 \\
(18.82)\end{array}$ & $\begin{array}{c}9.73 \\
(18.19)\end{array}$ & $\begin{array}{c}27.47 \\
(31.62)\end{array}$ & $\begin{array}{c}26.67 \\
(31.11)\end{array}$ & $\begin{array}{c}27.07 \\
(31.37)\end{array}$ & $\begin{array}{c}33.07 \\
(35.12)\end{array}$ & $\begin{array}{l}40.00 \\
(39.25)\end{array}$ & $\begin{array}{c}36.53 \\
(37.21)\end{array}$ & $\begin{array}{l}36.00 \\
(36.89)\end{array}$ & $\begin{array}{l}45.07 \\
(42.19)\end{array}$ & $\begin{array}{c}40.53 \\
(39.56)\end{array}$ & $\begin{array}{l}39.20 \\
(38.78)\end{array}$ & $\begin{array}{c}46.93 \\
(43.26)\end{array}$ & $\begin{array}{c}43.07 \\
(41.04)\end{array}$ \\
\hline $\begin{array}{c}\text { T5-Azoxystrobin } \\
250 \% \mathrm{SC}\end{array}$ & $\begin{array}{c}9.87 \\
(18.32)\end{array}$ & $\begin{array}{c}10.13 \\
(18.57)\end{array}$ & $\begin{array}{c}10.00 \\
(18.44)\end{array}$ & $\begin{array}{c}21.87 \\
(27.89)\end{array}$ & $\begin{array}{c}26.13 \\
(30.76)\end{array}$ & $\begin{array}{l}24.00 \\
(29.35)\end{array}$ & $\begin{array}{c}26.40 \\
(30.93)\end{array}$ & $\begin{array}{c}36.53 \\
(37.21)\end{array}$ & $\begin{array}{c}31.47 \\
(34.14)\end{array}$ & $\begin{array}{c}30.13 \\
(33.31)\end{array}$ & $\begin{array}{l}39.73 \\
(39.10)\end{array}$ & $\begin{array}{c}34.93 \\
(36.25)\end{array}$ & $\begin{array}{c}34.13 \\
(35.77)\end{array}$ & $\begin{array}{c}41.33 \\
(40.03)\end{array}$ & $\begin{array}{c}37.73 \\
(37.92)\end{array}$ \\
\hline T6-Propineb 70\% WP & $\begin{array}{c}9.60 \\
(18.06)\end{array}$ & $\begin{array}{c}9.33 \\
(17.80)\end{array}$ & $\begin{array}{c}9.47 \\
(17.93)\end{array}$ & $\begin{array}{l}23.47 \\
(28.99)\end{array}$ & $\begin{array}{l}25.60 \\
(30.41)\end{array}$ & $\begin{array}{c}24.53 \\
(29.71)\end{array}$ & $\begin{array}{l}28.00 \\
(31.96)\end{array}$ & $\begin{array}{l}33.07 \\
(35.12)\end{array}$ & $\begin{array}{c}30.53 \\
(33.56)\end{array}$ & $\begin{array}{c}32.53 \\
(34.79)\end{array}$ & $\begin{array}{l}40.80 \\
(39.72)\end{array}$ & $\begin{array}{c}36.67 \\
(37.29)\end{array}$ & $\begin{array}{c}36.53 \\
(37.21)\end{array}$ & $\begin{array}{c}49.07 \\
(44.49)\end{array}$ & $\begin{array}{c}42.80 \\
(40.88)\end{array}$ \\
\hline T7-Mancozeb 70\%WP & $\begin{array}{c}9.60 \\
(18.06)\end{array}$ & $\begin{array}{c}9.07 \\
(17.53)\end{array}$ & $\begin{array}{c}9.33 \\
(17.80)\end{array}$ & $\begin{array}{l}25.07 \\
(30.06)\end{array}$ & $\begin{array}{c}22.67 \\
(28.45)\end{array}$ & $\begin{array}{c}23.87 \\
(29.26)\end{array}$ & $\begin{array}{c}27.47 \\
(31.62)\end{array}$ & $\begin{array}{c}39.73 \\
(39.10)\end{array}$ & $\begin{array}{c}33.60 \\
(35.44)\end{array}$ & $\begin{array}{c}34.40 \\
(35.93)\end{array}$ & $\begin{array}{c}41.33 \\
(40.03)\end{array}$ & $\begin{array}{c}37.87 \\
(38.00)\end{array}$ & $\begin{array}{c}38.13 \\
(38.15)\end{array}$ & $\begin{array}{c}46.93 \\
(43.26)\end{array}$ & $\begin{array}{c}42.53 \\
(40.73)\end{array}$ \\
\hline $\begin{array}{c}\text { T8-Hexaconazole } 5 \% \\
\text { EC }\end{array}$ & $\begin{array}{c}9.87 \\
(18.32)\end{array}$ & $\begin{array}{c}9.60 \\
(18.06)\end{array}$ & $\begin{array}{c}9.73 \\
(18.19)\end{array}$ & $\begin{array}{c}18.13 \\
(25.22)\end{array}$ & $\begin{array}{l}17.60 \\
(24.82)\end{array}$ & $\begin{array}{c}17.87 \\
(25.02)\end{array}$ & $\begin{array}{c}19.73 \\
(26.39)\end{array}$ & $\begin{array}{c}23.47 \\
(28.99)\end{array}$ & $\begin{array}{l}21.60 \\
(27.71)\end{array}$ & $\begin{array}{l}20.80 \\
(27.15)\end{array}$ & $\begin{array}{l}26.40 \\
(30.93)\end{array}$ & $\begin{array}{l}23.60 \\
(29.08)\end{array}$ & $\begin{array}{l}22.13 \\
(28.08)\end{array}$ & $\begin{array}{l}35.73 \\
(36.73)\end{array}$ & $\begin{array}{c}28.93 \\
(32.56)\end{array}$ \\
\hline $\begin{array}{c}\text { T9-Propiconazole } \\
\text { 25\%EC }\end{array}$ & $\begin{array}{c}8.00 \\
(16.44)\end{array}$ & $\begin{array}{c}9.87 \\
(18.32)\end{array}$ & $\begin{array}{c}8.93 \\
(17.40)\end{array}$ & $\begin{array}{l}15.20 \\
(22.96)\end{array}$ & $\begin{array}{c}14.93 \\
(22.74)\end{array}$ & $\begin{array}{c}15.07 \\
(22.85)\end{array}$ & $\begin{array}{c}17.07 \\
(24.41)\end{array}$ & $\begin{array}{c}19.73 \\
(26.39)\end{array}$ & $\begin{array}{c}18.40 \\
(25.41)\end{array}$ & $\begin{array}{l}17.60 \\
(24.82)\end{array}$ & $\begin{array}{l}22.93 \\
(28.63)\end{array}$ & $\begin{array}{l}20.27 \\
(26.77)\end{array}$ & $\begin{array}{c}18.13 \\
(25.22)\end{array}$ & $\begin{array}{c}30.67 \\
(33.64)\end{array}$ & $\begin{array}{l}24.40 \\
(29.62)\end{array}$ \\
\hline $\begin{array}{l}\text { T10-Difenconzole } \\
25 \% \text { EC }\end{array}$ & $\begin{array}{c}9.33 \\
(17.80)\end{array}$ & $\begin{array}{c}10.40 \\
(18.82)\end{array}$ & $\begin{array}{c}9.87 \\
(18.32)\end{array}$ & $\begin{array}{l}17.60 \\
(24.82)\end{array}$ & $\begin{array}{c}16.53 \\
(24.00)\end{array}$ & $\begin{array}{c}17.07 \\
(24.41)\end{array}$ & $\begin{array}{c}18.93 \\
(25.81)\end{array}$ & $\begin{array}{c}22.67 \\
(28.45)\end{array}$ & $\begin{array}{l}20.80 \\
(27.15)\end{array}$ & $\begin{array}{l}20.00 \\
(26.58)\end{array}$ & $\begin{array}{c}26.13 \\
(30.76)\end{array}$ & $\begin{array}{l}23.07 \\
(28.72)\end{array}$ & $\begin{array}{l}20.53 \\
(26.96)\end{array}$ & $\begin{array}{l}31.20 \\
(33.97)\end{array}$ & $\begin{array}{c}25.87 \\
(30.59)\end{array}$ \\
\hline T11-Control & $\begin{array}{c}9.33 \\
(17.80)\end{array}$ & $\begin{array}{c}9.33 \\
(17.80)\end{array}$ & $\begin{array}{c}9.33 \\
(17.80)\end{array}$ & $\begin{array}{c}28.53 \\
(32.30)\end{array}$ & $\begin{array}{c}34.67 \\
(36.09)\end{array}$ & $\begin{array}{c}33.73 \\
(35.53)\end{array}$ & $\begin{array}{c}38.93 \\
(38.63)\end{array}$ & $\begin{array}{c}56.78 \\
(48.93)\end{array}$ & $\begin{array}{c}47.87 \\
(43.80)\end{array}$ & $\begin{array}{c}44.27 \\
(41.73)\end{array}$ & $\begin{array}{l}69.33 \\
(56.40)\end{array}$ & $\begin{array}{c}56.80 \\
(48.93)\end{array}$ & $\begin{array}{c}45.87 \\
(42.65)\end{array}$ & $\begin{array}{c}84.27 \\
(66.66)\end{array}$ & $\begin{array}{c}65.07 \\
(53.80)\end{array}$ \\
\hline S.Em \pm & NS & NS & NS & 1.06 & 0.87 & 0.71 & 1.14 & 0.86 & 0.86 & 1.16 & 1.32 & 0.91 & 0.87 & 1.49 & 0.90 \\
\hline $\mathrm{CD}$ at $5 \%$ & & & & 3.12 & 2.56 & 2.11 & 3.37 & 2.55 & 2.55 & 3.43 & 3.89 & 2.69 & 2.57 & 4.39 & 2.65 \\
\hline
\end{tabular}


Table.2 Field efficacy of fungicides on the yield of chrysanthemum

\begin{tabular}{|c|c|c|c|c|c|c|c|c|c|c|}
\hline \multirow[t]{2}{*}{ Treatments } & \multirow{2}{*}{$\begin{array}{l}\text { Conc. } \\
(\%)\end{array}$} & \multicolumn{3}{|c|}{ After V spray } & \multicolumn{3}{|c|}{ Per cent disease control } & \multirow{2}{*}{$\begin{array}{l}\text { Pooled } \\
\text { (kg/plot) }\end{array}$} & \multirow{2}{*}{$\begin{array}{l}\text { Yield } \\
\text { (t/ha) }\end{array}$} & \multirow[t]{2}{*}{$\mathrm{C}: \mathrm{B}$} \\
\hline & & 2016 & 2017 & Pooled & 2016 & 2017 & Pooled & & & \\
\hline T1-Tebuconazole & 0.1 & $\begin{array}{c}14.40 \\
(22.31)^{*}\end{array}$ & $\begin{array}{l}25.33 \\
(30.24)\end{array}$ & $\begin{array}{c}19.87 \\
(26.48)\end{array}$ & 31.47 & 58.93 & 45.20 & 5.27 & 8.78 & 4.13 \\
\hline $\begin{array}{l}\text { T2-Tebuconazole } 50 \%+ \\
\text { trifloxystrobin } 25 \% \mathrm{WG}\end{array}$ & 0.05 & $\begin{array}{l}16.00 \\
(23.59)\end{array}$ & $\begin{array}{c}28.00 \\
(31.96)\end{array}$ & $\begin{array}{l}22.00 \\
(27.99)\end{array}$ & 29.87 & 56.27 & 43.07 & 4.93 & 8.22 & 3.77 \\
\hline $\begin{array}{l}\text { T3-Azoxystrobin } 4.8 \%+ \\
\text { Chlorothalonil } 40 \%\end{array}$ & 0.1 & $\begin{array}{c}30.93 \\
(33.81)\end{array}$ & $\begin{array}{c}38.13 \\
(38.15)\end{array}$ & $\begin{array}{c}34.53 \\
(36.01)\end{array}$ & 14.93 & 46.13 & 30.53 & 3.90 & 6.50 & 3.02 \\
\hline T4-Chlorothalonil 75\% WP & 0.2 & $\begin{array}{c}39.20 \\
(38.78)\end{array}$ & $\begin{array}{c}46.93 \\
(43.26)\end{array}$ & $\begin{array}{c}43.07 \\
(41.04)\end{array}$ & 6.67 & 37.33 & 22.00 & 3.13 & 5.22 & 2.27 \\
\hline T5-Azoxystrobin $250 \% \mathrm{SC}$ & 0.1 & $\begin{array}{c}34.13 \\
(35.77)\end{array}$ & $\begin{array}{c}41.33 \\
(40.03)\end{array}$ & $\begin{array}{c}37.73 \\
(37.92)\end{array}$ & 11.73 & 42.93 & 27.33 & 3.83 & 6.39 & 2.64 \\
\hline T6-Propineb 70\% WP & 0.2 & $\begin{array}{c}36.53 \\
(37.21)\end{array}$ & $\begin{array}{c}49.07 \\
(44.49)\end{array}$ & $\begin{array}{c}42.80 \\
(40.88)\end{array}$ & 9.33 & 35.20 & 22.27 & 3.77 & 6.28 & 3.00 \\
\hline T7-Mancozeb 70\%WP & 0.2 & $\begin{array}{c}38.13 \\
(38.15)\end{array}$ & $\begin{array}{c}46.93 \\
(43.26)\end{array}$ & $\begin{array}{c}42.53 \\
(40.73)\end{array}$ & 7.73 & 37.33 & 22.53 & 3.37 & 5.61 & 2.69 \\
\hline T8-Hexaconazole 5\% EC & 0.1 & $\begin{array}{c}22.13 \\
(28.08)\end{array}$ & $\begin{array}{c}35.73 \\
(36.73)\end{array}$ & $\begin{array}{c}28.93 \\
(32.56)\end{array}$ & 23.73 & 48.53 & 36.13 & 4.83 & 8.06 & 3.92 \\
\hline T9-Propiconazole $25 \%$ EC & 0.1 & $\begin{array}{c}18.13 \\
(25.22)\end{array}$ & $\begin{array}{c}30.67 \\
(33.64)\end{array}$ & $\begin{array}{c}24.40 \\
(29.62)\end{array}$ & 27.73 & 53.60 & 40.67 & 4.87 & 8.11 & 3.88 \\
\hline T10-Difenconzole $25 \% \mathrm{EC}$ & 0.1 & $\begin{array}{c}20.53 \\
(26.96)\end{array}$ & $\begin{array}{c}31.20 \\
(33.97)\end{array}$ & $\begin{array}{c}25.87 \\
(30.59)\end{array}$ & 25.33 & 53.07 & 39.20 & 4.77 & 7.94 & 3.63 \\
\hline T11-Control & - & $\begin{array}{c}45.87 \\
(42.65)\end{array}$ & $\begin{array}{c}84.27 \\
(66.66)\end{array}$ & $\begin{array}{c}65.07 \\
(53.80)\end{array}$ & - & - & - & 3.00 & 5.00 & 2.47 \\
\hline S.Em \pm & & \multirow{2}{*}{$\begin{array}{l}0.87 \\
2.57\end{array}$} & \multirow{2}{*}{$\begin{array}{l}1.49 \\
4.39\end{array}$} & \multirow{2}{*}{$\begin{array}{l}0.90 \\
2.65\end{array}$} & - & - & - & 0.08 & - & - \\
\hline $\mathrm{CD}$ at $5 \%$ & & & & & - & - & - & 0.25 & - & - \\
\hline
\end{tabular}


Further percent disease reduction over control (PDC) was calculated for all treatments and presented in the Table 2. Among ten treatments during 2016, 2017 and pooled data highest PDC of 31.47, 58.93, 45.20 respectively on leaves was in tebuconazole at 0.1 per cent followed by tebuconazole + trifloxystrobin at $0.05(29.87,56.93$ and 45.20 respectively).

\section{Yield}

Yield obtained in fungicides treated plots indicated that, highest and significant yield of 8.78 tonnes per ha was recorded in tebuconazole at 0.1 per cent concentration treated plot (Table 2) followed by tebuconazole + trifloxystrobin 25 per cent WG $(0.05 \%)$ with a yield of 8.22 tonns/ha on par with T9-propiconazole (8.11t/ha). The lowest yield of 5.00 tonns per ha, obtained in untreated control plot.

But from the farmer's point of view, the economics of disease management is important. In the present investigation tebuconazole $(0.1 \%)$ has given highest total returns, net returns and additional returns over control than any other fungicides. Tebuconazole 50 per cent + trifloxystrobin 25 per cent WG $(0.05 \%)$ was next in order with respect to all the 3 above mentioned parameters. However, tebuconazole and hexaconazole has recorded highest incremental benefit: cost (4.13 and 3.92 respectively). Propiconazole with 3.88 remained as next best fungicide. The results are in agreement with the findings of several workers; Anonymous (2002b) reported propiconazole and iprodione were effective fungicides against Alternaria blight of sunflower. In the present study, mancozeb did not prove well. However mancozeb was reported to be effective fungicide against A.helianthi in sunflower (Wadiphasme et al., 1991 and Amaresh, 1997). Mancozeb spray at 30,45 and 60 DAS could give good control of Alternaria blight of sunflower (Anon., 2002b). AICRP on sunflower centres at different parts of India reported propiconazole (Anon., 2003), while Mesta et al., (2003), Arun kumar (2008) and Devaraja (2011) reported hexaconazole as effective fungicide against Alternaria blight.

In conclusion, two years results revealed that Tebuconazole $(0.1 \%)$ and tebuconazole $50 \%$ + trifloxystrobin $25 \% \mathrm{WG}(0.05 \%)$ were effective in minimizing the per cent disease index and getting higher yields.

\section{References}

Alfieri, S. A., 1968, Septoria leaf spot of Chrysanthemum. Pl. Pathol. Circula., 73.

Amaresh, Y. S., 1997, Studies on foliar diseases of sunflower (Helianthus annuus L.) with special reference to Alternaria leaf blight caused by Alternaria helianthi (Hansf.) Tubaki and Nishihara. M.Sc. (Agri.) Thesis, Univ. Agric. Sci., Dharwad.

Anonymous, 2002b, DOR Research Highlights 1997-2000. Directorate of Oilseeds Research, Hyderabad: 70-75.

Anonymous, 2003, Annual Progress Report of Sunflower 2002-03. Directorate of Oilseeds Research, Hyderabad, p. 6166.

Anonymous, 2016, Information on area and production of chrysanthemum in Karnataka state.

Arun Kumar, G. S., 2008, Studies on leaf blight of chrysanthemum caused by Alternaria alternata (Fr.) Keissler. $M$. Sc. (Agri.) Thesis, Univ. Agric. Sci., Dharwad.

Cook, R. T. A., 2001, First report in England of changes in the susceptibility of Puccinia horiana, the cause of chrysanthemum white rust to triazole and strobilurin fungicides. Pl. Pathol, 
50:792.

Devaraja, O., 2011, Investigations on chrysanthemum leaf blight caused by Alternaria alternata (Fr.) Keissler., $M$. Sc. (Agri.) Thesis, Univ. Agric. Sci., Dharwad.

Ghosh, P. P., Mandal, D., Laha, S. and Dasgupta, M. K., 2009, Dynamics and severity model in managing fungal diseases. J. Pl. Prot. Sci. 1(1): 55 - 59.

Kher, M. A., 1990, Chrysanthemum "Queen of the East'. Indian Hort., 35(1): 10-13.

Luong, T. M., Huynh, L. M. T., Hoang, H. M. T., Tesoriero, L. A., Burgers, L. W., Phan, H. T. and Davies, P., 2010, First report of Pythium root rot of Chrysanthemum in Vietnam and control with metalaxyl drench. Australasian Pl. Dis. Notes., 5:51- 54.

Mesta, R.K., Sunkad, G. and Katti, P., 2003, Chemical control of Alternaria blight of sunflower. Extended Summaries of National Seminar on Stress Management in Oilseeds for attaining self reliance in vegetable oils, January 28 - 30, held at DOR, Hyderabad, p. 149-151

Mridha, M. A. U., Jabbar, F., Bhuiyan, M. K., Rahman, M., Akter, F. and Dewan, S., 2007, The severity and cause of leaf spot disease of Pongamia pinnata L. and fungicidal control of the pathogen. J. Fore. Res., 18(3): 236-240.

NHB, 2014, All India area and production of Horticultural crops, Indian Horticulture Database.

Nishi, N., Muta, T., Ito, Y., Nakamura, M. and Tsukiboshi, T., 2009, Ray speck of Chrysanthemum caused by Stemphylium lycopersici in Japan. J. General Pl. Pathol. 75: 80 - 82.

Schmidt, 1958, A fungal leaf spot disease of Chrysanthemum caused by Alternaria chrysanthemi sp. nov. Pflanzenchutz., 21: 13-20.

Sikdar, A. K. and Krishnaswami, S., 1980, Assessment of leaf yield loss of two Mulberry varieties due to leaf spot disease. Indian J. Seri., 19: 9 - 12.

Strider, D. L., 1985, Fusarium wilt of Chrysanthemum: Pathogen - free rooted cuttings and susceptibility of new cultivars. Pl. Dis., 69: 836-838.

Wadiphasme S. S., Ingole, O. Y., Raut, N.K. and Asalmdle, M. N., 1991, Chemical control of leaf spot caused by Alternaria helianthi on sunflower. Indian J. Pl. Prot., 19: 203-205.

Wheeler, B. E. J., 1969, An introduction to plant diseases. John Wiley and Sons Ltd., London

\section{How to cite this article:}

Divyajyothi, U., Suresh D. Ekabote, H. Narayanswamy, P. Narayanaswamy, H. Ravindra and Satish, K.M. 2018. Evaluation of New Fungicides Molecules against Leaf Blight of Chrysanthemum caused by Alternaria spp.. Int.J.Curr.Microbiol.App.Sci. 7(07): 3884-3890. doi: https://doi.org/10.20546/ijcmas.2018.707.452 